\title{
Diagnostik
}

\section{Schmerzskala für Demenzkranke} vorgestellt

\author{
Die üblichen Schmerzskalen sind bei schwerer Demenz häufig nicht \\ geeignet. Forscher der Universität Bamberg haben jetzt ein Werkzeug \\ entwickelt, das Schmerzen solcher Patienten möglichst objektiv ermit- \\ teln soll.
}

W ie und in welchem Ausmaß Patienten mit fortgeschrittener Demenz Schmerzen empfinden, lässt sich nur schwer sagen. Einiges ist zumindest aus der Forschung bekannt: „Die Schmerzmatrix wird unspezifischer, Schmerzreize werden eher stärker und das Gehirn kann solche Reize nicht mehr ausreichend dämpfen", erläuterte Prof. Stefan Lautenbacher auf dem Geriatriekongress in Frankfurt am Main. Die Annahme, dass mit dem Verlust der Kognition wenigstens auch die Schmerzwahrnehmung zurückgehe, sei wohl falsch: „Der Schmerz nimmt sehr wahrscheinlich nicht ab, er kann nur nicht

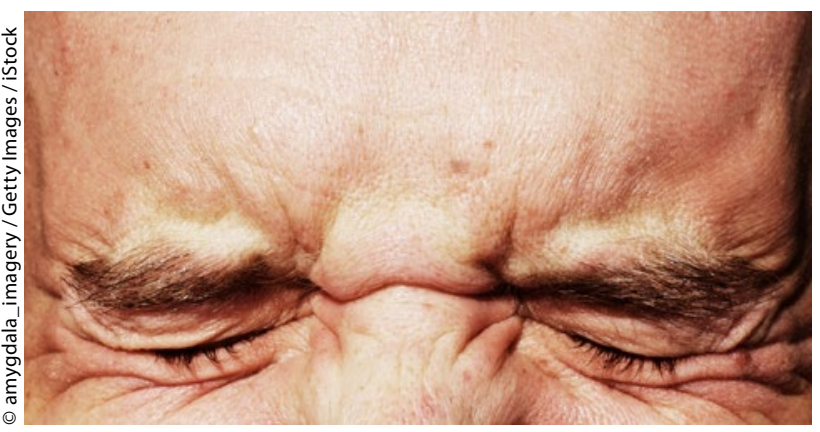

Deutlicher Hinweis auf starke Schmerzen. skala: Hier werden die Patienten schlicht gefragt, ob sie keine, leichte, mäßige, starke oder unerträglich starke Schmerzen haben. Die Antworten würden mit zunehmender Demenz jedoch immer unzuverlässiger. Letztlich, so der Exper-
Demenzkranke genauer angeschaut, möglichst viele relevante Aspekte herausgezogen und im Kranken- sowie Pflegealltag getestet. Herausgekommen ist die Skala „Pain Assessment in Impaired Cognition (PAIC-15)“. Sie bildet jeweils fünf Aspekte zu Gesichtsausdruck, Körperbewegung und Lautäußerungen ab. Bei der Mimik wird etwa geschaut, ob Patienten die Augenbrauen zusammenziehen, die Augen zusammenkneifen oder die Oberlippe hochziehen. Eine kurze Erklärung veranschaulicht, was genau damit gemeint ist. Das soll für mehr Objektivität sorgen.

Bei den Körperbewegungen stehen Unruhe, Abwehr von Pflegemaßnahmen und Aufsuchen einer Schutzhaltung im Vordergrund, bei der Vokalisation wird erfasst, ob die Patienten laut schreien, tiefe unartikulierte Laute von sich geben oder Schmerzwörter wie etwa "Aua“, „Oh Weh“ oder „Autsch“ äußern.

Für jeden Aspekt gibt es 0 mehr richtig kommuniziert werden“, erläuterte der Leiter der Abteilung Physiologische Psychologie an der Universität Bamberg. Eine gute Schmerztherapie sei daher auch bei Demenzkranken wichtig.

\section{Übliche Skalen für}

\section{Demenzpatienten ungeeignet}

Übliche Instrumente wie die visuelle Analogskala oder die Gesichtsskala aus der Pädiatrie mit lächelnden bis traurigen Gesichtern seien daher ungeeignet. Erstere sei selbst für kognitiv Gesunde eine Herausforderung, Letztere könnten Demenzkranke häufig nicht mehr richtig interpretieren. Am ehesten eigne sich bei beginnenden bis mittleren kognitiven Beeinträchtigungen die Kategorie- te, sind Therapeuten auf eine genaue Fremdbeobachtung von Körperhaltung, Mimik und Lautäußerungen angewiesen.

Inzwischen existierten zwar etliche Tools, die solche nonverbalen Äußerungen zur Schmerzerfassung heranziehen, ein Problem sei jedoch ein sehr hoher Interpretationsspielraum. Begriffe wie "schmerzvoller Ausdruck “, „leerer Blick“ oder „verängstigtes Aussehen“ spiegelten vor allem die Wahrnehmung des Beobachters wider und seien daher nur wenig objektiv, so der Schmerzforscher.

\section{Schmerzskala mit 15 Aspekten}

Ein internationales Team um Lautenbacher hat sich mithilfe eines EU-geförderten Programms die Schmerzskalen für bis 3 Punkte (nicht, gering, mäßig, stark), die Skala reicht also bis maximal 45 Punkte. Mit einer solchen Skala lasse sich abschätzen, ob Demenzkranke unter Schmerzen leiden, und ein Stück weit auch, wie stark. Allerdings könnten Ärzte damit kaum herausfinden, was weh tue. Hier sei noch weitere Forschung nötig, sagte Lautenbacher.

Erhältlich ist der PAIC-15 über die Universität Bamberg, Professur für Physiologische Psychologie.

Thomas Müller

29. Jahreskongress der Deutschen Gesellschaft für Geriatrie (DGG), 28-30. September, Frankfurt am Main. Symposium S212: Schmerz und Psyche im Alter. 